\title{
Las Competencias de Salud Global para Estudiantes de Enfermería: Percepciones de Docentes en América Latina
}

\section{Global Health Competencies among Nursing Students: Perceptions of Teachers in Latin America}

\section{As Competências de Saúde Global para Estudantes de Enfermagem: Percepções de Docentes na América Latina}

\author{
L. Wilson"a, L. Morán-Peñaa, ${ }^{b}$, R. Zárate-Grajales ${ }^{b}$, I.A. Costa- \\ ${\text { Mendesc, C.A. Arena- Ventura', I. Tami-Maury }{ }^{\text {, }} \text {, N. Warrene }}^{\text {e }}$ \\ ${ }^{2}$ Escuela de Enfermería, Universidad de Alabama en Birmingham, Birmingham, Alabama, Estados Unidos \\ bEscuela Nacional de Enfermería y Obstetricia, Universidad Nacional Autónoma de México, Ciudad de México, \\ México \\ 'Escuela de Enfermería de Ribeirão Preto de la Universidad de São Paulo, Brasil \\ ¿Departamento de Ciencias de Comportamiento, Centro de Cáncer M. D. Anderson Universidad de Texas, Hous- \\ ton, Texas, Estados Unidos \\ Escuela de Enfermería, Universidad Johns Hopkins, Baltimore, Maryland, Estados Unidos
}

Recibido: 2 mayo 2017

Aceptado: 20 enero 2018

\section{Resumen}

Objetivo: El propósito de este estudio fue describir las percepciones de docentes de enfermería de habla hispana en América Latina, sobre las competencias relacionadas a la salud global que deben mostrar los estudiantes de enfermería del pregrado.

Métodos: Este estudio descriptivo fue basado en una muestra de docentes de escuelas de enfermería miembros de la Asociación Latinoamericana de Escuelas y Facultades de Enfermería (ALADEFE) y de la Asociación de Escuelas de la Zona Centro Sur de México, las cuales recibieron un correo electrónico con una liga para responder una encuesta electrónica por Survey Monkey®C. La encuesta incluyó una lista de 30 competencias en salud global dividida en seis dimensiones. Los docentes indicaron en una escala Likert de 4 puntos la relevancia de cada competencia para la educación de enfermería en el nivel de pregrado $(1=$ Completamente en desacuerdo; $2=$ En desacuerdo; $3=$ De acuerdo; $4=$

*Autor para correspondencia. Correo electrónico: eneolm@yahoo.com (L. Morán-Peña) 
Completamente de acuerdo). Resultados: En total, 110 profesores de nueve países respondieron a la encuesta. El promedio de cada ítem fue entre 3.0 - 4.0, esto indica que los profesores estuvieron de acuerdo en que todas las competencias son relevantes para la formación de estudiantes de enfermería a nivel de pregrado.

Conclusiones: Los resultados de este estudio sugirieron que estas competencias deben ser incluidas en los currículos de enfermería a nivel de pregrado, para formar a los estudiantes en su labor como enfermeras y enfermeros en un mundo globalizado y prepararlos para contribuir a la Cobertura y el Acceso Universal a la Salud (Salud Universal).

Palabras clave: Salud global; educación en enfermería; competencias; Región América.

\section{Abstract}

Objective: To describe the perceptions of Spanish-speaking nursing teachers in Latin America on the global health competences which, undergraduate nursing students should demonstrate.

Methods: This descriptive study was based on a sample of teachers of nursing schools belonging to the Latin American Association of Nursing Schools and Faculties, and the Association of Schools of the Center-South Zone in Mexico. These institutions received an invitation via e-mail to respond to a survey through the Survey Monkey(C) platform. The survey included a list of 30 Global Health competencies grouped in 6 dimensions. Teachers indicated through a 4-point Likert scale their perception on the relevance to undergraduate nursing education of each of these competencies $(1=$ total disagreement, $2=$ disagreement, $3=$ agreement, $4=$ total agreement).

Results: In total, 110 professors of 9 countries responded to the survey. The average score per item was $3.0-4.0$ suggesting that the teachers agreed that all competencies are relevant to the formation of undergraduate nursing students.

Conclusions: These results suggested that these competencies should be part of the objectives of undergraduate nursing curricula in order to prepare the students to contribute to the Coverage and Access to Universal Health agenda.

Keywords: Global health; nursing, education; competencies; Americas Region.

\section{Resumo}

Objetivo: O propósito deste estudo foi descrever as percepções de docentes de enfermagem de fala hispana na América Latina, sobre as competências relacionadas à saúde global que devem mostrar os estudantes de enfermagem de graduação.

Métodos: Este estudo descritivo foi baseado em uma amostra de docentes de escolas de enfermagem membros da Associação Latino-americana de Escolas e Faculdades de Enfermagem (ALADEFE) e da Associação de Escolas da Zona Centro Sul do México, as quais receberam um e-mail electrónico com

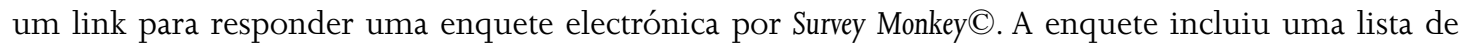
30 competências em saúde global dividida em seis dimensões. Os docentes indicaram em uma escala Likert de 4 pontos a relevância de cada competência para a educação de enfermagem no nível de graduação ( 1 = Completamente em discordância; $2=$ Em discordância; $3=$ Em concordância; $4=$ Completamente em concordância).

Resultados: Em total, 110 professores de nove países responderam à enquete. A média de cada item foi entre 3.0-4.0, isto indica que os professores concordaram em que todas as competências são relevantes para a formação de estudantes de enfermagem a nível de graduação. Conclusões: Os resultados deste estudo sugeriram que estas competências devem ser incluídas nos currículos de enfermagem a nível de graduação, para formar aos estudantes em seu labor como enfermeiras e enfermeiros em um mundo globalizado e prepará-los para contribuir à Cobertura e o Acesso Universal à Saúde (Saúde Universal). 
Palavras chave: Saúde global; educação em enfermagem; competências; Região América.

\section{Introducción}

Como consecuencia de la globalización se han generado cambios sociales, políticos y económicos a nivel mundial, regional y local que han influido en la salud global. Las enfermedades infecciosas como Zika, VIH, Ebola, pueden traspasar fronteras fácilmente. También la globalización de negocios y cambios en las formas de comunicación han influenciado los estilos de vida, incluidos los de alimentación y nutrición, que desencadenan enfermedades crónicas. Los efectos del cambio climático, el aumento de la población global y la escasez de recursos pueden influir en la salud de nuestro planeta ${ }^{1}$.

En el marco internacional, la Organización Mundial de Salud (OMS) y la Organización Panamericana de Salud (OPS) han identificado como una prioridad la promoción de la Cobertura y el Acceso Universal a la Salud (Salud Universal) ${ }^{2-4}$, a la par, los estados miembros de las Naciones Unidas han aprobado un plan para el Desarrollo Sostenible como una guía del desarrollo global para el período 2015-2030 $0^{5}$. Estos nuevos escenarios implican la necesidad de asegurar que los estudiantes de enfermería tengan las competencias necesarias para la práctica de salud en un mundo globalizado.

En este contexto, se consideró indispensable, en primer lugar, delimitar cuales deberían ser estas competencias; con este propósito, educadores de enfermería han explorado las competencias globales de salud para enfermeras en las Américas ${ }^{6}$ y África ${ }^{7}$, el medio ha sido un estudio que se ha replicado en diversos países: Estados Unidos, Canadá, el Caribe y América Latina, para identificar las percepciones de los docentes de enfermería sobre las competencias relacionadas a salud global que deben mostrar los estudiantes de enfermería de pregrado ${ }^{6}$. En dichos estudios, los investigadores adaptaron una lista de competencias en salud global que fue desarrollada para estudiantes de medicina ${ }^{8}$. La lista final incluyó 30 competencias, distribuidas en seis dimensiones; cada competencia se evalúa con una escala tipo Likert en la que el puntaje fue: 1 = Completamente en desacuerdo; 2 = En desacuerdo; 3 = De acuerdo; 4 = Completamente de acuerdo (Figura No. 1). En cada estudio se envió invitación para participar en la encuesta en línea a docentes de enfermería. En el primer estudio la muestra por conveniencia se constituyó por docentes de todas las escuelas de enfermería en los Estados Unidos, Canadá y el Caribe de habla inglés, y más de 600 docentes de enfermería en América Latina y el Caribe de habla española y portuguesa. Un total de 542 profesores contestaron la encuesta en inglés y 51 en español ${ }^{6}$. Asimismo, la invitación a responder la encuesta fue enviada a todas las escuelas de enfermería en Brasil, se recibieron 222 respuestas de los docentes que aceptaron'. En África contestaron 63 docentes (49 en inglés, 12 en francés, y 2 en portugués) ${ }^{7}$. El promedio de las respuestas a cada competencia en estos tres estudios fue entre 3.0 - 4.0, lo que sugirió que los docentes estaban de acuerdo en que estas competencias son esenciales para estudiantes en enfermería a nivel pregrado.

Sin embargo, el trabajo realizado en las Américas estaba limitado por una baja representación de profesores de enfermería en los países de habla hispana, lo que limitó la capacidad para identificar prioridades en la región y representó una dificultad para lograr un consenso internacional. Por lo tanto, el propósito de este estudio fue identificar las percepciones de docentes de enfermería sobre las competencias relacionadas a la salud global, que deben mostrar los estudiantes de enfermería del pregrado en países de América Latina de habla hispana. El estudio es una réplica realizada con el apoyo de la Asociación Latinoamericana de Escuelas y Facultades de Enfermería (ALADEFE), y de la Asociación de Escuelas y Facultades de Enfermería de la Región Centro Sur de México, así como de algunos Centros Colaboradores de Enfermería y Partería de OPS de Estados Unidos, México y Brasil.

Para los propósitos de este documento, salud global se define como "un área de estudio, investigación y práctica que le da prioridad al mejoramiento de la salud y al logro de equidad en salud para toda la población a nivel mundial. La salud global enfatiza los asuntos, determinantes y soluciones de salud trasnacionales; implica muchas disciplinas dentro y más allá de las ciencias de la salud y promueve la colaboración interdisciplinaria; y es una síntesis de la prevención basada en la población con 
cuidado clínico a nivel individual” ${ }^{10}$. Koplan et al. ${ }^{10}$ posicionan la Salud Global, como una extensión del dominio disciplinar de la salud pública con respecto al escenario geográfico de sus operaciones, y la diferencian de la salud universal en cuanto a que ésta limita el objetivo de la salud pública hacia el desarrollo de programas de prevención en las poblaciones, mientras que a la Salud Global le conciernen todas las estrategias posibles dirigidas a mejorar la salud de las poblaciones.

\section{Métodos}

Se realizó un estudio transversal con educadores de enfermería en países de América Latina de habla hispana. Para ello, se reclutó la muestra a través de correo electrónico enviado por la Presidenta y Vicepresidentas regionales de la ALADEFE a directivos de las escuelas y facultades de enfermería miembros de dicha Asociación. La invitación incluyó los objetivos y alcances del estudio y las instrucciones para responder la encuesta en la plataforma Survey Monkey ${ }^{\circ}$. La encuesta incluyó las 30 competencias distribuidas en seis dimensiones, tal como fue utilizada en los estudios anteriores ${ }^{6,7,9}$ (Figura 1). De igual manera, en el caso de México se envió la invitación para participar a la Asociación de Facultades y Escuelas de Enfermería de la Región Centro Sur del país, se remitió la información a todas las directoras de las 22 escuelas pertenecientes a la misma. La muestra fue por conveniencia de los investigadores, se incluyeron aquellos instrumentos completos de los profesores que decidieron participar voluntariamente.

\section{Resultados}

En total, 110 docentes de 366 que fueron invitados respondieron a la encuesta (24 decanas, 81 profesores y 6 personas con otras responsabilidades académicas). Los países representados fueron Argentina $(\mathrm{n}=22)$, Bahamas $(\mathrm{n}=1)$, Chile $(\mathrm{n}=14)$, Colombia $(\mathrm{n}=17)$, República Dominicana $(\mathrm{n}=1)$, México $(\mathrm{n}=18)$, Panamá $(\mathrm{n}=22)$, Perú $(\mathrm{n}=12)$ y Uruguay $(\mathrm{n}=3)$. Aun cuando 110 docentes respondieron a la primera parte de la encuesta correspondiente a los datos demográficos, solamente entre 86 - 89 contestaron a las preguntas relacionadas a las 30 competencias sobre salud global. El número de respuestas para cada renglón está indicado en la Figura No. 1; en ésta se presentan agrupadas por dimensiones las 30 competencias con el promedio y la desviación estándar para cada una de ellas. La confiabilidad de las seis dimensiones fue evaluada con alpha de Cronbach, su resultado se brinda al final de cada dimensión. La confiabilidad de 5 de las 6 dimensiones fue entre $0.76-0.87$, lo que representa una consistencia interna adecuada ${ }^{12}$. Sólo una dimensión obtuvo una confiabilidad menor de 0.70 (Carga global de la enfermedad =0.60), el cual fue el valor mínimo utilizado para determinar una consistencia interna adecuada ${ }^{12}$. Un factor que pudo contribuir a esta baja confiabilidad es que la dimensión tiene solamente tres posibles respuestas.

El promedio de cada ítem fue entre 3.0 - 4.0, indicativo de que los docentes estuvieron de acuerdo, que todas las competencias son relevantes para los estudiantes de enfermería a nivel de pregrado.

Figura I. Promedio y desviación estándar de cada competencia y dimensión

1: Carga global de la enfermedad. Una comprensión básica de la carga global de enfermedad representa una parte esencial de una educación en enfermería moderna. Este conocimiento es fundamental para participar en discusiones de establecimiento de prioridades, racionamiento de cuidados y financiación de la salud e investigaciones en salud. Un graduado en enfermería debe lograr: 
1a. Describir las causas principales de morbilidad y mortalidad a nivel

$3.6(.52)$

mundial, y cómo el riesgo de enfermedad varía de región a región.

1b. Describir los esfuerzos importantes de la salud pública para reducir

disparidades en salud global (tales como los Objetivos del Desarrollo del

Milenio y el Fondo Global de Lucha contra el SIDA, TB, y Malaria).

$3.6(.52)$

1c. Discutir la fijación de prioridades, razonamiento de cuidados médicos

y financiamiento de salud e investigaciones sanitarias.

(Alpha de Cronbach = .60) (Promedio/Desviación Estándar: 3.55/.39) $(\mathrm{n}=89)$

2: Implicaciones de salud por migración, viajes y reubicación. El manejo apropiado de las necesidades de los pacientes, conforme a las perspectivas y los riesgos involucrados en los viajes internacionales y en el nacimiento de un niño en el extranjero. Un graduado en enfermería debe lograr:

\begin{tabular}{|c|c|}
\hline Ítem & Promedio (DE) \\
\hline $\begin{array}{l}\text { 2a. Demostrar una comprensión sobre los riesgos para la salud que implican } \\
\text { los viajes internacionales y el nacimiento en el extranjero. }\end{array}$ & $3.3(.62)$ \\
\hline $\begin{array}{l}\text { 2b. Reconocer cuando un viaje o el lugar de nacimiento en el extranjero pone al paciente } \\
\text { en riesgo de enfermedades inusuales o de presentaciones inusuales de enfermedades } \\
\text { comunes, para así lograr hacer una evaluación o referencia apropiada. }\end{array}$ & $3.3(.67)$ \\
\hline 2c. Describir cómo el contexto cultural influye en la percepción de la salud y de la enfermedad. & $3.8(.42)$ \\
\hline $\begin{array}{l}\text { 2d. Ser capaz de percibir las preocupaciones individuales de } \\
\text { salud de una manera culturalmente sensible. }\end{array}$ & $3.7(.49)$ \\
\hline 2e. Comunicarse efectivamente con los pacientes y sus familias por medio de un intérprete. & $3.7(.54)$ \\
\hline $\begin{array}{l}\text { 2f. Identificar las regiones del mundo y/o actividades de viaje asociadas al } \\
\text { riesgo creciente de enfermedades peligrosas para la vida, incluidos VIH/ } \\
\text { SIDA, malaria y tuberculosis resistente a drogas múltiples. }\end{array}$ & $3.4(.56)$ \\
\hline Alpha de Cronbach $=.80)($ Promedio/Desviación Estándar: 3.5/.39) $(\mathrm{n}=89)$ & \\
\hline
\end{tabular}

3: Determinantes sociales y ambientales de la salud. Los factores sociales, económicos y ambientales son determinantes importantes de la salud; además, la salud es más que simplemente la ausencia de enfermedad. Los enfermeros deben comprender como las condiciones sociales, económicas y ambientales inciden en la salud, para así reconocer factores de riesgo para enfermedades en sus pacientes y contribuir para mejoras en la salud pública. Un graduado en enfermería debe lograr:

\begin{tabular}{ll}
\hline Ítem & Promedio (DE) \\
\hline $\begin{array}{l}\text { 3a. Describir cómo situaciones sociales y económicas tales como pobreza, } \\
\text { educación y estilos de vida afectan la salud y el acceso al cuidado médico. }\end{array}$ & $3.8(.37)$ \\
\hline $\begin{array}{l}\text { 3b. Enumerar los determinantes sociales importantes de salud y su impacto } \\
\text { en las diferencias de esperanza de vida entre y dentro de los países. }\end{array}$ & $3.7(.47)$ \\
\hline $\begin{array}{l}\text { 3c. Describir el impacto de un ingreso bajo, educación y factores de } \\
\text { comunicación en el acceso y calidad del cuidado médico. }\end{array}$ & $3.7(.48)$ \\
\hline $\begin{array}{l}\text { 3d. Describir la relación entre el acceso al agua potable, sistema de aguas residuales/ } \\
\text { cloacas, alimento, calidad del aire y la salud del individuo y la población. }\end{array}$ & $3.7(.47)$ \\
\hline $\begin{array}{l}\text { 3e. Describir la relación entre la degradación ambiental y la salud humana. } \\
\text { (Alpha de Cronbach = .83) (Promedio/Desviación Estándar: 3.7/.34) }(\mathrm{n}=89)\end{array}$ & $3.7(.48)$ \\
\hline
\end{tabular}


4: Globalización de la salud y del cuidado médico* . La globalización está cambiando profundamente los patrones de las enfermedades y la disponibilidad de profesionales de la salud alrededor del mundo. Además del efecto directo de las enfermedades, de los profesionales de la salud y de los pacientes que se mueven por el mundo, los acuerdos e instituciones globales influyen en la capacidad de gobiernos y sistemas de salud para atender a las necesidades de salud de sus poblaciones. Un graduado en enfermería debe lograr:

\begin{tabular}{|c|c|}
\hline Ítem & Promedio (DE) \\
\hline $\begin{array}{l}\text { 4a. Analizar cómo las tendencias globales en las prácticas de cuidado médico, el comercio y } \\
\text { la cultura, los acuerdos multinacionales y las organizaciones multinacionales contribuyen a la } \\
\text { calidad y a la disponibilidad de la salud y del cuidado médico a nivel local e internacional. }\end{array}$ & $3.4(.59)$ \\
\hline $\begin{array}{l}\text { 4b. Describir diversos modelos nacionales para la prestación pública y/o privada de } \\
\text { cuidado médico y sus efectos respectivos sobre gasto en salud y cuidado médico. }\end{array}$ & $3.4(.64)$ \\
\hline $\begin{array}{l}\text { 4c. Analizar cómo los viajes y el comercio contribuyen a la } \\
\text { propagación de enfermedades comunicables y crónicas. }\end{array}$ & $3.3(.60)$ \\
\hline $\begin{array}{l}\text { 4d. Analizar tendencias generales e influencias en la disponibilidad y } \\
\text { movimiento global de los trabajadores del cuidado médico. }\end{array}$ & $3.3(.63)$ \\
\hline 4e. Describir la disponibilidad y escasez nacional y global del trabajador del cuidado médico. & $3.3(.64)$ \\
\hline $\begin{array}{l}\text { 4f. Describir los patrones más comunes de migración del trabajador del cuidado } \\
\text { médico y su impacto en la disponibilidad del cuidado de salud en el país de } \\
\text { donde el trabajador del cuidado médico sale y el país al cual él emigra. }\end{array}$ & $3.1(.64)$ \\
\hline$($ Alpha de Cronbach = .87) $($ Promedio/Desviación Estándar: 3.3/.48) $(\mathrm{n}=89)$ & \\
\hline
\end{tabular}

5: Cuidado médico en lugares de bajos recursos. Las necesidades de cuidado médico y los recursos disponibles son muy diferentes entre locales con pocos y muchos recursos. Para cuidar de los pacientes con eficacia, independientemente de la disponibilidad de recursos en lo local, un graduado en enfermería debe lograr:

\begin{tabular}{ll}
\hline Ítem & Promedio (DE) \\
\hline $\begin{array}{l}\text { 5a. Determinar las barreras de salud y cuidado médico en lugares de } \\
\text { bajos recursos tanto a nivel local como internacional. }\end{array}$ & $3.6(.62)$ \\
\hline $\begin{array}{l}\text { 5b. Demostrar una comprensión de los asuntos culturales y } \\
\text { éticos al trabajar con poblaciones en desventaja. }\end{array}$ & $3.7(.47)$ \\
\hline $\begin{array}{l}\text { 5c. Demostrar la capacidad de adaptar habilidades y prácticas clínicas en } \\
\text { los lugares en donde existen pocos recursos disponibles. }\end{array}$ & $3.8(.40)$ \\
\hline $\begin{array}{l}\text { 5d. Identificar los signos y síntomas de las enfermedades comunes más importantes para } \\
\text { facilitar el diagnóstico en caso de no contar con pruebas avanzadas que a menudo son } \\
\text { inasequibles en lugares de bajos recursos (enfermedad cardiovascular, cáncer y diabetes). }\end{array}$ & $3.7(.52)$ \\
\hline $\begin{array}{l}\text { 5e. Describir el papel del manejo sindrómico y los algoritmos } \\
\text { clínicos para el tratamiento de enfermedades comunes. }\end{array}$ & $3.6(.58)$ \\
\hline $\begin{array}{l}\text { 5f. Identificar las intervenciones clínicas y las estrategias integradas que han demostrado } \\
\text { mejorar substancialmente la salud del individuo y/o de la población en lugares de bajos } \\
\text { recursos (ej.: inmunizaciones, drogas esenciales, programas materno-infantiles). }\end{array}$ & $3.7(.52)$ \\
\hline $\begin{array}{l}\text { 5g. Los estudiantes que eligen participar en lugares de bajos recursos lejos de su hogar, deberán } \\
\text { demostrar que ellos han tenido un entrenamiento que los prepare para esta elección. } \\
\text { (alpha de Cronbach = .77) (Promedio/Desviación Estándar: 3.7/.48) (n = 89) }\end{array}$ & $3.6(.58)$ \\
\hline
\end{tabular}

6: Salud como derecho humano y recurso de desarrollo. Los derechos humanos inciden en la salud de los individuos y de las poblaciones. La salud también es un elemento esencial del desarrollo económico y social. Para advocar con eficacia por la salud de los pacientes y de las comunidades, con base en la

\footnotetext{
* El término cuidado médico fue retomado como aparece en el instrumento original, considerándolo para este estudio como sinónimo de cuidado a la salud, ya que de acuerdo a la definición de Salud Global de Koplan y colaboradores incluida en la introducción, éste incorpora a las diversas disciplinas del área de la salud.
} 
comprensión de la relación entre los derechos humanos, el desarrollo socioeconómico y la salud, un graduado en enfermería debe lograr:

\begin{tabular}{ll}
\hline Ítem & Promedio (DE) \\
\hline 6a. Demostrar una comprensión básica de la relación entre salud y los derechos humanos. & $3.7(.45)$ \\
\hline $\begin{array}{l}\text { 6b. Demostrar familiaridad con organizaciones y los acuerdos que tratan sobre los } \\
\text { derechos humanos en relación al cuidado médico y la investigación médica. }\end{array}$ & $3.6(.58)$ \\
\hline $\begin{array}{l}\text { 6c. Describir el papel de la Organización Mundial de la Salud (OMS) al enlazar la salud y los derechos } \\
\text { humanos, la Declaración Universal de los Derechos Humanos, las Pautas Éticas Internacionales para la } \\
\text { Investigación Biomédica que involucra Seres Humanos (2002) y la Declaración de Helsinki (2008). }\end{array}$ & $3.7(.48)$ \\
\hline (Alpha de Cronbach $=.76)$ (Promedio/Desviación Estándar: 3.6/.41) (n=89) & \\
\hline
\end{tabular}

Nota: La lista de competencias fue publicada originalmente en Wilson L, Harper DC, Tami-Maury I, et al. Competencias mundiales de salud para enfermeras en las Américas. J Prof Nurs. 2012; 28(4): 199-258, con permiso de Elsevier.

En total, 65 docentes escribieron comentarios cualitativos y éstos fueron ya analizados y reportados en un artículo ex profeso, en conjunto con los comentarios en inglés y portugués ${ }^{11}$.

La Tabla 1 presenta los promedios y desviaciones estándar de las seis dimensiones utilizadas en este estudio, comparados con los obtenidos en los estudios anteriores en América Latina ${ }^{6}$ y África ${ }^{7}$. Esta tabla también indica la confiabilidad de las seis dimensiones en los estudios anteriores. La confiabilidad de la primera dimensión (Carga Global de la Enfermedad) fue más alta en los estudios anteriores (entre 0.68 - 0.81), sin embargo, la confiabilidad de las otras dimensiones fue similar a la confiabilidad del presente estudio.

Tabla 1. Comparación de puntuación en las dimensiones entre estudios previos y la Réplica de ALADEFE

\begin{tabular}{|c|c|c|c|c|}
\hline Región & $\mathrm{N}$ & Media & $\mathrm{DE}$ & $\begin{array}{l}\text { Alpha de } \\
\text { Cronbach }\end{array}$ \\
\hline \multicolumn{5}{|c|}{ Dimensión 1: Carga global de la enfermedad } \\
\hline América de habla inglesa ${ }^{6}$ & 567 & 3.3 & .61 & .81 \\
\hline América de habla hispana ${ }^{6}$ & 51 & 3.5 & .70 & .78 \\
\hline Brasil $^{9}$ & 222 & $\mathrm{NA}^{*}$ & NA & .75 \\
\hline África ${ }^{7}$ & 63 & 3.4 & .70 & .68 \\
\hline $\begin{array}{l}\text { América de habla } \\
\text { hispana Réplica } \\
\text { de ALADEFE }\end{array}$ & 110 & 3.6 & 39 & 60 \\
\hline \multicolumn{5}{|c|}{ Dimensión 2: Implicaciones de salud por migración, viajes y reubicación } \\
\hline América de habla inglesa ${ }^{6}$ & 567 & 3.5 & .46 & .84 \\
\hline América de habla hispana ${ }^{6}$ & 51 & 3.4 & .74 & .92 \\
\hline Brasil $^{9}$ & 222 & NA & NA & .79 \\
\hline África ${ }^{7}$ & 63 & 3.3 & .60 & .86 \\
\hline $\begin{array}{l}\text { América de habla } \\
\text { hispana Réplica } \\
\text { de ALADEFE }\end{array}$ & 110 & 3.5 & .39 & .80 \\
\hline \multicolumn{5}{|c|}{ Dimensión 3: Determinantes sociales y ambientales de la salud } \\
\hline América de habla inglesa ${ }^{6}$ & 567 & 3.6 & .49 & .89 \\
\hline América de habla hispana ${ }^{6}$ & 51 & 3.6 & .66 & .96 \\
\hline Brasil $^{9}$ & 222 & NA & NA & .83 \\
\hline África ${ }^{7}$ & 63 & 3.6 & .51 & .89 \\
\hline
\end{tabular}




\begin{tabular}{|c|c|c|c|c|}
\hline $\begin{array}{l}\text { América de habla } \\
\text { hispana Réplica } \\
\text { de ALADEFE }\end{array}$ & 110 & 3.7 & .34 & .83 \\
\hline \multicolumn{5}{|c|}{ Dimensión 4: Globalización de la salud y del cuidado médico } \\
\hline América de habla inglesa ${ }^{6}$ & 567 & 3.0 & .56 & .89 \\
\hline América de habla hispana ${ }^{6}$ & 51 & 3.3 & .69 & .94 \\
\hline Brasil $^{9}$ & 222 & NA & NA & .87 \\
\hline África ${ }^{7}$ & 63 & 3.3 & .57 & .90 \\
\hline $\begin{array}{l}\text { América de habla } \\
\text { hispana Réplica } \\
\text { de ALADEFE }\end{array}$ & 110 & 3.3 & .48 & .87 \\
\hline \multicolumn{5}{|c|}{ Dimensión 5: Cuidado médico en lugares de bajos recursos } \\
\hline América de habla inglesa ${ }^{6}$ & 567 & 3.4 & .47 & .84 \\
\hline América de habla hispana ${ }^{6}$ & 51 & 3.6 & .59 & .93 \\
\hline Brasil $^{9}$ & 222 & NA & NA & .81 \\
\hline África $^{7}$ & 63 & 3.5 & .55 & .86 \\
\hline $\begin{array}{l}\text { América de habla } \\
\text { hispana Réplica } \\
\text { de ALADEFE }\end{array}$ & 110 & 3.7 & .48 & .77 \\
\hline \multicolumn{5}{|c|}{ Dimensión 6: Salud como derecho humano y recurso de desarrollo } \\
\hline América de habla inglesa ${ }^{6}$ & 567 & 3.3 & .55 & .82 \\
\hline América de habla hispana ${ }^{6}$ & 51 & 3.5 & .66 & .91 \\
\hline Brasil $^{9}$ & 222 & NA & NA & .68 \\
\hline África ${ }^{7}$ & 63 & 3.5 & .49 & .82 \\
\hline $\begin{array}{l}\text { América de habla } \\
\text { hispana Réplica } \\
\text { de ALADEFE }\end{array}$ & 110 & 3.6 & .41 & .76 \\
\hline
\end{tabular}

* En el estudio replicado en Brasil los autores no reportaron el promedio y DS de las seis dimensiones. Solamente reportaran el promedio y DS de cada ítem ${ }^{25}$

\section{Discusión}

La primera dimensión de competencias describe la carga global de enfermedades, como conocimiento fundamental para discusiones sobre prioridades, otorgamiento de cuidados, financiación de la salud y el desarrollo de investigaciones en la salud. Entre las dimensiones, ésta fue la que obtuvo la media más baja. La carga global de enfermedades abarca los datos internos de salud de los distintos países, por lo que el riesgo de enfermedad varía de región a región y permite analizar cómo son influenciados por direccionamientos externos, especialmente esfuerzos para la reducción de las disparidades en salud global. En este aspecto, es importante enfatizar que las inequidades en salud entre e intra países son inaceptables y no pueden ser solucionadas con acciones restringidas a medidas técnicas del sector salud, o solamente circunscritas al ámbito nacional, una vez que requieren soluciones políticas globales. Actores estatales y no estatales deben estar más conectados por un diálogo político trasparente en los procesos de toma de decisiones que afectan a la salud ${ }^{13}$. Lo anterior también permite identificar la necesidad de que en la formación de los estudiantes de enfermería se enfatice cómo en el diagnóstico y planeación de intervenciones deberá considerarse el contexto, ya que éste se revela mutable, contradictorio, tensionado por las fuerzas sociales que representan, por un lado, un escenario conservador $y$, por otro, los cambios y las rupturas a favor de un nuevo proyecto para combatir las desigualdades. Es precisamente en el interior de este contexto complejo que actúan los agentes de las prácticas sociales de salud ${ }^{14}$. 
La segunda dimensión tiene seis ítems relacionados al manejo apropiado de las necesidades de los pacientes, en el cual se consideran las perspectivas y los riesgos involucrados en los viajes internacionales y en el nacimiento de un niño en el extranjero. La educación en enfermería necesita considerar en sus currículos el movimiento creciente de personas y profesionales de salud y sus consecuencias, para el trabajo de los profesionales de enfermería. Los patrones de migración crean retos no solamente para los trabajadores de salud, sino también para los países que los reciben ${ }^{15}$. Las enfermeras y enfermeros deben reconocer los riesgos de salud involucrados en los viajes internacionales, así como ser capaces de percibir las preocupaciones individuales de salud y comunicarse de una manera culturalmente sensible. La sensibilidad cultural debe ser parte de la enseñanza de enfermería, especialmente en salud global, como parte fundamental para la comprensión de los retos que se presentan en el cuidado a la salud en situaciones de diversidad ${ }^{16}$. Así, el enfermero debe construir habilidades interpersonales de comunicación basada en empatía y aceptación. Estas dimensiones se relacionan directamente y son detalladas en la cuarta dimensión que enfoca la globalización de la salud y el cuidado médico. Cuestiones como los patrones de migración de los trabajadores de salud, el comercio, la cultura y los acuerdos internacionales influyen en las condiciones de salud los cuales deben estar presentes en la formación de los profesionales de enfermería, en tanto que el cuidado como un acto comunicativo requiere de conocimientos científicos y tecnológicos, pero también del contexto cultural donde viven, se recrean y enferman los sujetos cuidados ${ }^{17}$.

Asociaciones de Escuelas de Enfermería constituidas por la zona geográfica a la que pertenecen, como por ejemplo ALADEFE, y las correspondientes a los países, constituyen poderosos entes dinamizadores para iniciar discusiones y cambios en los currículos, como la inserción de temas de salud global, cobertura universal de salud y el rol del profesional de enfermería, vivencias reportadas, así como las estrategias adoptadas que pueden iluminar los caminos y fortalecer la concepción de redes de educación en enfermería. En este contexto, es fundamental que los líderes exploren al máximo su diversidad de modo constructivo, simultáneamente, consolidar una identidad de red con foco en la interacción y colaboración entre sus miembros. Esto implica también la revisión de modelos educativos y reorientación de los planes de estudio hacia modelos sociales complejos, así como un análisis permanente del impacto de la globalización ${ }^{18}$.

Los factores sociales, económicos y ambientales que afectan a la salud son descritos en la tercera dimensión. De este modo, los enfermeros se deben preocupar en describir cómo situaciones sociales y económicas como pobreza, educación y estilos de vida, bajo ingreso económico, acceso al agua potable y la degradación ambiental afectan la salud y el acceso de las personas a los cuidados de salud. Es imperativo enfrentar los retos de salud y desarrollo que traspasan a los diferentes grupos de países y direccionan la discusión sobre la agenda de desarrollo para la salud, con enfoque central en los recursos humanos de salud ${ }^{19}$. La Comisión de las Naciones Unidas para el Empleo en Salud y Crecimiento Económico ofrece una oportunidad sin precedentes para el compromiso político, que explora y formula recomendaciones sobre cómo las inversiones en recursos humanos representan una estrategia crítica para luchar contra las inequidades en el acceso de los trabajadores de salud, así como estimular la creación de empleos en sectores de salud y sociales para un desarrollo inclusivo ${ }^{20}$. Un abordaje más holístico debe incluir la planeación de los recursos humanos para la salud dentro de contextos más amplios de los sistemas social y de salud, el desarrollo de estos planes, implica soluciones en diferentes niveles y la necesidad de colaboración mutua entre gobiernos, investigadores, administradores de sistemas de salud, legisladores y profesionales de salud ${ }^{21}$.

La quinta dimensión trata específicamente de las necesidades de cuidado médico en lugares de bajos recursos, en el cual se aspira al cuidado de los pacientes con eficacia. Además, es fundamental desarrollar estrategias que apoyen la creación de puentes entre los sectores públicos, no gubernamentales y privados para minimizar las barreras de acceso a los servicios de salud en áreas con bajos recursos ${ }^{22}$. 
Son incluidos también aspectos éticos que deben ser considerados cuando hay grandes diferencias de poder económico entre los involucrados en el proceso de cuidado a la salud.

La sexta dimensión enfatiza la salud como derecho humano y recurso para el desarrollo. Estas competencias permiten que los enfermeros y enfermeras adopten un lenguaje universal común basado en los principios de los derechos humanos y de la salud como derecho. Además, del reconocimiento internacional de que la globalización puede contribuir a estos derechos humanos y al desarrollo, la realidad es que los derechos humanos son constantemente subordinados a dogmas del fundamentalismo de mercado con énfasis en el lucro y no en el desarrollo sostenible ${ }^{23}$. De esta forma, un discurso explícito sobre la importancia de los derechos humanos es necesario y debería ser incluido en los Objetivos de Desarrollo Sostenible, para conseguir la búsqueda de cambios que aseguren la cobertura universal de salud en los diferentes países ${ }^{24}$. En este sentido, el conocimiento de los derechos humanos es condición para que los enfermeros puedan también abogar por los derechos de la población, que muchas veces no se reconoce como sujeto de derechos ${ }^{25}$.

La enseñanza en salud global posibilitará el desarrollo de competencias claves para el cuidado humanizado en salud, el aprendizaje basado en la práctica, el mejoramiento de habilidades de comunicación y el profesionalismo ${ }^{26}$. Los promedios de las dimensiones e ítems fueron similares a los datos de los estudios anteriores ${ }^{6,7,9}$. Mientras que el alpha de Cronbach de las seis dimensiones fue más bajo en confiabilidad que en los otros estudios, sólo una dimensión tuvo una confiabilidad menor de 0.70 (Carga global de la enfermedad $=0.60)$ (vea Figura 1$)$. A pesar de sus limitaciones, este estudio contribuye al diálogo sobre las competencias, lo cual resulta indispensable para una actuación responsable y comprometida de los profesionales de enfermería en salud global.

\section{Conclusiones}

Este estudio destaca la necesidad imperativa de integrar programas de formación sobre salud global al currículo de la carrera de enfermería, con el objetivo de preparar futuros enfermeros y enfermeras capaces de influenciar las prácticas sanitarias y de educación en salud, no solamente a nivel nacional, sino también en un plano global.

Los hallazgos de este estudio, basados en las respuestas de 110 docentes miembros de la Asociación Latinoamericana de Escuelas y Facultades de Enfermería y de una Asociación regional de México, deben ser considerados como un esfuerzo inicial para determinar los conocimientos, las habilidades y las destrezas en salud global que deben delinear los programas educativos dirigidos a los estudiantes de pregrado de la carrera de enfermería en América Latina y otras regiones del mundo.

Los resultados del estudio confirman la necesidad de continuar el diálogo entre los docentes y líderes de enfermería latinoamericanos, para establecer las competencias mínimas esenciales en salud global a considerarse en los programas de pregrado en enfermería y así armonizar el currículo de esta disciplina entre los distintos países de la región.

La coherencia entre estos hallazgos y los de otras regiones sugiere un consenso de que la importancia de estas competencias trasciende las fronteras geográficas y los niveles de recursos. Este consenso, sin embargo, es un primer paso. Se necesitan esfuerzos adicionales para delinear cómo estas competencias pueden ser operacionalizadas en los currículos existentes.

Los educadores ahora pueden centrarse en cómo integrar estas competencias en el currículo de acuerdo al nivel académico del estudiante. Esto podría variar dependiendo de los aspectos educativos, culturales y del sistema de salud específicos del ambiente en el cual las enfermeras son preparadas.

\section{Responsabilidades éticas}

Protección de personas y animales. Los autores declaran que para esta investigación no se han realizado experimentos en seres humanos ni en animales. 
Confidencialidad de los datos. Los autores declaran que han seguido los protocolos de sus centros de trabajo sobre la publicación de datos de los participantes: docentes, decanas y personas con otras responsabilidades académicas dentro de las Escuelas o Facultades de Enfermería participantes en el estudio.

Derecho a la privacidad y consentimiento informado. Los autores han obtenido el consentimiento informado de los sujetos referidos en el artículo. Al tratarse de una encuesta en línea, los participantes estuvieron anticipados en las instrucciones de llenado de su derecho a no responderlo si así lo consideraban, o a retirarse de la encuesta en el momento que lo desearan, y que el llenado completo del mismo implicaba su consentimiento informado.

\section{Financiamiento: Ninguno}

Conflicto de Intereses: Los autores declaran no tener conflicto de intereses.

Agradecimientos: A la Asociación Latinoamericana de Escuelas y Facultades de Enfermería, la Asociación de Escuelas y Facultades de Enfermería Región Centro Sur de México, a los Centros Colaboradores de Enfermería: Escuela Nacional de Enfermería y Obstetricia de la UNAM y Escuela de Enfermería de la Universidad de Alabama en Birmingham; Escuela de Enfermería de la Universidad de Sao Paulo en Riberao Preto Brasil; y Escuela de Enfermería de la Universidad Johns Hopkins.

\section{Referencias}

1. Horton R, Beaglehole R, Bonita R, et al. From public to planetary health: a manifesto. Lancet. 2014; 383(9920): 847. https://doi.org/10.1016/S0140-6736(14)60409-8

2. Organización Panamericana de la Salud. Organización Mundial de la Salud. 53. ${ }^{\circ}$ Consejo Directivo. 66.a Sesión del Comité Regional de la OMS para las Américas. Estrategia para el acceso universal a la salud y la cobertura universal de salud. Washington: OPS-OMS; 2014.

3. Pan American Health Organization. World Health Organization. Universal Health Coverage. OPSOMS; 2016.

4. World Health Organization. Research for universal health coverage: World health report 2013. WHO; 2013.

5. United Nations. Sustainable development goals. 17 goals to transform our world. New York: UN; 2016.

6. Wilson L, Harper DC, Tami-Maury I, et al. Global health competencies for nurses in the Americas. J Prof Nurs. 2012; 28(4): 213-22. http://dx.doi.org/10.1016/j.profnurs.2011.11.021

7. Warren N, Breman R, Budhathoki C, et al. Perspectives of nursing faculty in Africa on global health nursing competencies. Nurs Outlook. 2015; 64(2): 179-85.

http://dx.doi.org/10.1016/j.outlook.2015.11.016

8. Arthur MA, Battat R, Brewer TF. Teaching the basics: core competencies in global health. Infect Dis Clin North Am. 2011; 25(2): 347-58. http://dx.doi.org/10.1016/j.idc.2011.02.013

9. Arena-Ventura CA, Costa-Mendes IA, Law-Wilson L, et al. Global health competencies from the perspective of nursing faculty from Brazilian higher education institutions. Rev. Latino-Am. Enfermagem. 2014; 22(2): 179-86. http://dx.doi.org/10.1590/0104-1169.2996.2400

10. Koplan JP, Bond TC, Merson MH, et al. Towards a common definition of global health. Lancet. 2009; 373(9679): 1993-5. https://dx.doi.org/10.1016/S0140-6736(09)60332-9

11. Wilson L, Moran L, Zarate R, et al. Qualitative description of global health nursing competencies by nursing faculty in Africa and the Americas. Rev. Latino-Am. Enfermagem. 2016; 24(2697): 1-10. http://dx.doi.org/10.1590/1518-8345.0772.2697

12. Polit DF, Beck CT. Nursing research: Generating and assessing evidence for nursing practice. 10th ed. Philadelphia: Lippincott Williams \& Wilkins; 2016.

13. Gill S, Benatar S. Global Health Governance and Global Power: A Critical Commentary on the Lancet-University of Oslo Commission Report. Int J Health Serv. 2016; 46(2): 346-65. 
http://dx.doi.org/10.1177/0020731416631734

14. De Almeida AH, Baldini Soares C. Educación en salud: análisis de la enseñanza en la graduación en enfermería. Rev. Latino-Am. Enfermagem. 2011; 19(3): 1-8.

15. Shaffer FA, Bakhshi M, To D, et al. Code for ethical international recruitment practices: the CGFNS alliance case study. Hum Resour Health. 2016; 14(Suppl 1): 113-9.

https://dx.doi.org/10.1186/s12960-016-0127-6

16. Costa-Mendes IA, Arena-Ventura CA, Trevizan MA, et al. Educación, liderazgo y colaboraciones: potencialidades que la enfermería puede dar a la Cobertura Universal de Salud. Rev. Latino-Am. Enfermagem. 2016; 24(e2673). 1-9. http://dx.doi.org/10.1590/1518-8345.1092.2673

17. Costa-Mendes IA, Arena-Ventura CA. Leading and advocating for global health competencies: a nursing challenge. J Nurs Manag. 2016; 24(7): 843-4. http://dx.doi.org/10.1111/jonm.12432

18. Ibarra-Mendoza TX, González JS. Competencia cultural: Una forma humanizada de ofrecer Cuidados de Enfermería. Index Enferm. 2006; 15(55): 44-8.

19. Campbell J, Buchan J, Cometto G, et al. Human resources for health and universal health coverage: fostering equity and effective coverage. Bull World Health Organ. 2013; 91(11): 853-63.

http://dx.doi.org/10.2471/BLT.13.118729

20. World Health Organization. Health workforce. High-Level Commission on Health Employment and Economic Growth. Geneva:WHO; 2016.

21. Shamian J, Tomblin G, Elliott A, et al. Human resources for health: a new narrative. Lancet. 2015; 386(9988): 25-6. https://dx.doi.org/10.1016/S0140-6736(15)61195-3

22. International Confederation of Midwives. International Council of Nurses. World Health Organization. Triad Communiqué 17 May 2014. Geneva: ICM. ICN. WHO; 2014.

23. United Nations Human Rigths. Independent Expert on the promotion of a democratic and equitable international order. Geneva: UNHR; 2012.

24. Williams C, Blaiklock A. Human Rights Discourse in the Sustainable Development Agenda Avoids Obligations and Entitlements; Comment on "Rights Language in the Sustainable Development Agenda: Has Right to Health Discourse and Norms Shaped Health Goals?”. IJHPM. 2016; 5(6): 387-90. http://dx.doi.org/10.15171/ijhpm.2016.29

25. Arena-Ventura CA, Falleiros-de Mello D, Dully-Andrade R, et al. Alianza de la enfermería con el usuario en la defensa del SUS. Rev Bras Enferm. 2012; 65(6): 893-8.

http://dx.doi.org/10.1590/S0034-71672012000600002

26. Tsai A, Fricchione G, Walensky R, et al. Global health training in US graduate psychiatric education. Acad Psychiatry. 2014; 38(4): 426-32. 